\title{
The effect of vowel similarity and syllable length on acoustic memory
}

\author{
LAURA L. HALL and SHEILA E. BLUMSTEIN \\ Brown University, Providence, Rhode Island 02912
}

\begin{abstract}
Darwin and Baddeley (1974) argue that the presence of recency, suffix, and modality effects are not attributable to the acoustic properties of the stimuli but, rather, to the acoustic distance between the items comprising the test series. The present study is designed to determine whether stimulus duration is a significant variable in acoustic memory. Eight different blocks of synthetic stimuli were prepared; one block each of $60 \mathrm{msec}$ similar and dissimilar syllables and $190 \mathrm{msec}$ similar and dissimilar syllables. The other four blocks consisted of these same vocabularies, but each list in the block had an eighth syllable suffix of the same duration as the syllables in the block. Significant recency and suffix effects are seen for dissimilar syllables of both durations. No effects are demonstrated for $60-\mathrm{msec}$ similar syllables, but both of the effects are seen for 190-msec similar syllables. These results indicate that whether or not a speech sound is preserved in precategorical acoustic storage (PAS) depends upon not only the acoustic distance between the stimuli, but also on other characteristics intrinsic to the stimuli, e.g., stimulus duration.
\end{abstract}

Crowder has conducted extensive studies investigating the role of auditory memory in speech perception. His original work employed a paradigm that required the subject to recall a list of seven digits in the correct order immediately following their presentation (Crowder \& Morton, 1969). This technique gives rise to three different effects exhibited by acoustic memory: the recency effect, the modality effect, and the suffix effect. The recency effect entails an advantage for the terminal item of the list. The modality effect is characterized by an advantage of auditory over visual presentation for the items at the end of the stimulus list. The suffix effect is observed when a redundant suffix, which the subject does not have to recall, is added to the end of the list. If the suffix is physically similar to the list items (in the speaker's voice, amplitude, etc.), both the recency and modality effects are abolished.

Crowder and Morton (1969) have proposed a theory of acoustic memory to account for the three observed effects. Although both visual and auditory input eventually lead to comparable forms of representation in the central short-term memory (STM), there are more peripheral processes taking place which carry information in prelinguistic form. Crowder and Morton refer to the auditory memory as precategorical acoustic storage (PAS). They suggest that the stimuli are stored for a short period of time in PAS before entering the central short-term memory. The recency effect arises from the fact that the last items presented remain in the store and are most easily retrievable. PAS retains information longer than the analogous visual precategorical store, thus accounting for the modality effect. It is char- acterized by limited space as well as a-limited time capacity, and the suffix effect is attributable to this space limitation. The verbal suffix writes over the final list item, thus eliminating the recency effect.

Crowder's later work included measuring the memory effects for lists of synthetic speech syllables. It was found that the modality, recency, and suffix effects did not occur if syllables differing in initial stop consonants (bah,dah,gah) were used. However, syllables differing in a final vowel (bee, bih, boo) gave the various effects. Crowder (1971) concluded that PAS is selective as to which material it retains: vowel information is retained, while consonant information is not.

An additional variable that seems to affect memory is stimulus duration. Crowder (1973a) has shown that recall impairment caused by a verbal suffix is greater for $300-\mathrm{msec}$ vowels than for $50-\mathrm{msec}$ vowels. This result suggests that vowels of a greater duration are represented in auditory memory better and for a longer time than are short vowels.

Darwin and Baddeley (1974) have offered an alternative explanation for the presence of acoustic memory effects in certain contexts and their absence in others. They argue that the three effects are not attributable to the acoustic characteristics of the stimuli (e.g., syllable durations or a consonantvowel distinction), but rather to the acoustic distance between the items comprising the test series. They question results of previous psychological studies that indicate the existence of a special processor for certain speech sounds, arguing that these findings may simply be a consequence of the particular stimulus set used. Darwin and Baddeley stress that an 
important factor that has been hitherto neglected in these studies is whether the stimulus sets are comprised of similar vowels or of dissimilar vowels. Similarity is specified in terms of the vowels' proximity in acoustic space.

Darwir and Baddeley's study demonstrated that neither lists of similar consonants (bah,dah,gah) nor similar vowels (bi,bae,be) displayed significant recency or suffix effects, while lists of dissimilar vowels (bi,bae,bu) and consonants (sha,ma,ga) did. As a result of this finding, they suggest that PAS retains a tape-recorder-like representation of all incoming sounds that is subject to degradation with time. This degradation may leave the memory trace underlying finer auditory discriminations less effective than that underlying coarser discriminations.

Based on this assumption, one would expect to find no acoustic memory effects for lists of similar syllables, regardless of the length of each stimulus. Conversely, lists of dissimilar syllables would display all of the effects regardless of stimulus length. Darwin and Baddeley included in their study both similar and dissimilar lists of $60-\mathrm{msec}$ syllables as well as dissimilar lists of 190 -msec syllables. As predicted, the lists of similar syllables displayed no memory effects, while the dissimilar lists demonstrated all of these effects.

Their study did not, however, include lists of long similar syllables. A strict interpretation of their model would suggest that no acoustic memory effects will be demonstrated for these lists of stimuli. However, if the various effects were to be found for these lists, it would be clear that stimulus similarity is not the only significant variable in acoustic memory.

The present study is designed specifically to examine the effect of syllable duration on acoustic memory and ultimately to test the validity of the tape-recorder hypothesis as presented by Darwin and Baddeley. This study includes the three conditions found in the Darwin and Baddeley study along with a fourth condition, lists of 190 -msec similar syllables. Each of these four conditions appears both with and without a suffix ending. The tape-recorder model predicts that all lists of dissimilar syllables should display acoustic memory effects, while similar lists should not. If these results are not obtained, one must question the tape-recorder hypothesis and consider the role of stimulus length on PAS.

\section{METHOD}

\footnotetext{
Subjects

Thirty students attending Brown University served as listeners in this study. All subjects were native speakers of English and had no known hearing impairment. All subjects had had no previous experience in hearing synthetic speech.
}

\section{Stimuli}

The test stimuli consisted of 10 synthetic $\mathrm{CV}$ syllables generated on a parallel resonance synthesizer at the Research Laboratory of Electronics at Massachusetts Institute of Technology. These stimuli were comprised of an initial consonant transition equivalent to the consonant $/ b /$ followed by the vowels $/ \mathrm{i} /$, $/ \mathrm{ae} /, / \mathrm{e} /, / \mathrm{u} /$ and $/ 3 /$. Starting frequencies for the initial consonant transition remained the same for all stimuli at $200 \mathrm{~Hz}$ (F1), $900 \mathrm{~Hz}$ (F2), and 2,000 $\mathrm{Hz}(\mathrm{F} 3)$.

Steady-state formant frequencies for the vowels comprising the test stimuli are displayed diagramatically in Figure 1. All sounds have additional steady third, fourth, and fifth formants at $2,500,3,500$, and $4,500 \mathrm{~Hz}$, respectively. In Figure 1 , the crosses indicate the similar vocabulary (bi,be,bae) and the circles, the dissimilar vocabulary (bi,be,bu).

Stimulus syllables were of two lengths, 60 and $190 \mathrm{msec}$. Syllables of both durations consisted of an initial $30-\mathrm{msec}$ consonant transition followed by steady-state frequencies of either 30 or $160 \mathrm{msec}$.

Test trials were composed of seven randomly ordered syllables from a vocabulary of either acoustically similar or acoustically dissimilar syllables. A stimulus suffix /by/ was added as eighth syllable to one-half of the test series. Stimuli were separated into eight blocks of 25 trials each, in which the vocabulary, length, and suffix (present in four of the eight blocks) remained constant. Four of the stimulus blocks included vocabularies of 190 msec similar syllables, 60 -msec similar syllables, 190 -msec dissimilar syllables, and 60 -msec dissimilar syllables. The other four blocks consisted of these same four vocabularies, but each list in the block had an eighth syllable suffix of the same duration as the syllables in that block. Each subject heard all eight blocks. The order of presentation of the blocks was different for each group of subjects, thus counterbalancing for a possible order effect.

\section{Procedure}

All subjects were tested in two separate 45 -min sessions. They received four blocks of trials in each session. Subjects were instructed to listen to the entire list of syllables and then to write the list down as quickly as possible in the order in which the stimuli were presented on the answer sheet provided. If the subject could not remember the entire list of seven syllables, he was

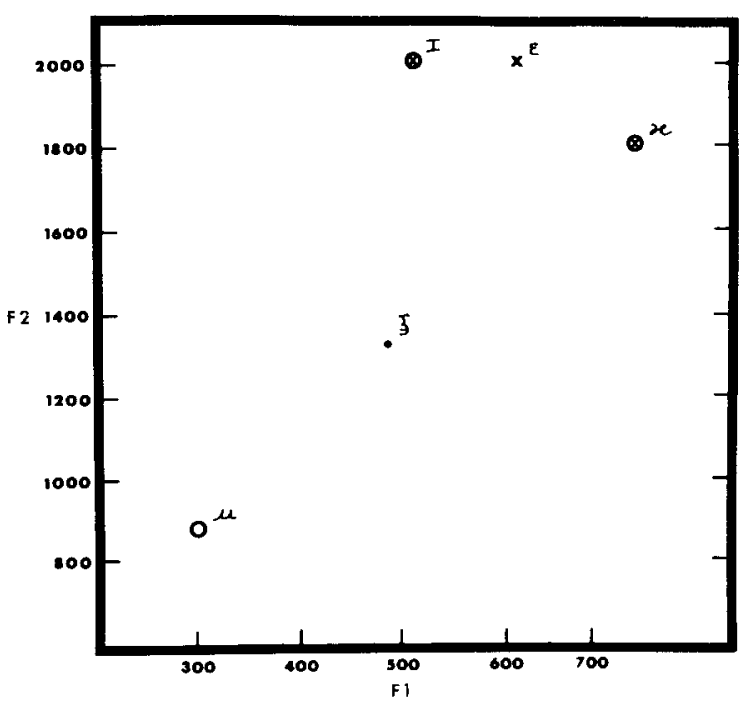

Figure 1. Frequency values of the first and second formants for stimuli used in the experiment. 
told to either guess at the syllables or leave the space for those syllables on the answer sheet blank. It was stressed that all lists had to be written down in the same order in which they were presented. The subjects were told, prior to the commencement of each block of trials, whether or not the lists contained an eighth syllable suffix. If there was a suffix, the subjects were instructed to write down only the first seven syllables and ignore the eighth.

The symbols that the subjects were to use to identify each syliable were written at the top of each answer sheet. An "i" was used to represent the syllable $/ \mathrm{bi} /$, an "a" represented the syllable /bae/, an "e"' was used to represent /be/, and a "u" was used for the syllable /bu/. Each block of trials was preceded by several repetitions of the syllables to be heard in the block. The subjects could hear these syllables as many times as necessary to become familiarized with the different syllables. As soon as the subjects felt confident with the sounds, the trials began.

The lists were played binaurally over headphones at a rate of 2 items/sec, with $12 \mathrm{sec}$ allowed for written recall. Each trial was preceded by a $500-\mathrm{Hz}$ warning tone. All blocks contained a total of 25 test trials, of which the first 5 were treated as practice and were not scored. The subjects were told prior to the onset of the experiment that the first 5 trials were practice.

\section{RESULTS}

The results for the present experiment are shown in Figure 2. The data were treated by a repeated measures analysis of variance for four factors: position, length, suffix, and similarity. Significant main effects were obtained for position $[F(1,29)=49.61$, $\mathrm{p}<.001]$, suffix $[\mathrm{F}(1,29)=67.62, \mathrm{p}<.001]$, and similarity $[\mathrm{F}(1,29)=119.33, \mathrm{p}<.001]$. An overall significant interaction was also obtained $[F(1,29)$ $=4.87, \mathrm{p}<.05]$. Subsequent post hoc comparisons based on the analysis of variance error term were conducted to ascertain recency and suffix effects for the various vocabularies. Recency effects for all vocabularies were determined by comparing the sixth and seventh serial positions. A significant decrease in errors for the seventh position denotes a recency effect. To determine the suffix effect, a comparison was made of the seventh serial position of each vocabulary containing a suffix to that same vocabulary without a suffix (which served as the control). ${ }^{1}$ A significant increase in errors noted for the vocabulary containing the suffix indicates a suffix effect.

Findings for vocabularies of dissimilar syllables of 60- and 190-msec durations replicate those of Darwin and Baddeley (1974). Significant recency effects are seen for both 190-msec dissimilar syllables [t(29) $=$ $3.35, \mathrm{p}<.01]$ and $60-\mathrm{msec}$ dissimilar syllables [t(29) $=4.07, \mathrm{p}<.01]$. In addition, highly significant suffix effects are found for these two conditions $[\mathrm{t}(29)=6.26, \mathrm{p}<.001 ; \mathrm{t}(29)=5.47, \mathrm{p}<.001$, respectively]. Also replicated are findings for vocabularies of similar syllables of a $60-\mathrm{msec}$ duration. These vocabularies display neither a recency effect nor a suffix effect $[\mathrm{t}(29)=1.11, \mathrm{p}>.05 ; \mathrm{t}(29)=$ $1.79, \mathrm{p}>.05$, respectively]. However, $190-\mathrm{msec}$ similar syllables display a significant recency effect $[t(29)=3.5, p<.01]$ as well as a highly significant suffix effect $[t(29)=6.23, p<.001]$. The presence of these two acoustic memory effects of vocabularies of $190-\mathrm{msec}$ similar syllables indicates that syllable length is a significant variable in acoustic memory.

\section{DISCUSSION}

The tape-recorder model for acoustic memory as described by Darwin and Baddeley (1974) asserts that auditory input is held in acoustic memory for a short period of time, during which the representation becomes degraded. The result of this degradation is to blur the information so that there may be sufficient information left to distinguish between a number of very different items but not enough left to distinguish between the same number of more similar ones.

The present study investigated whether an item's preservation in PAS is determined solely by the similarity or dissimilarity of the stimulus set or whether stimulus duration also plays a significant role in this determination. Darwin and Baddeley included, in their study, vocabularies of both 60-msec similar and $60-\mathrm{msec}$ dissimilar syllables as well as $190-\mathrm{msec}$ dissimilar syllables. The present study included these same three conditions as well as a fourth, 190-msec similar syllables. If stimulus duration plays no role in acoustic memory, then one should not find significant recency or suffix effects for the latter vocabulary. Results for the first three conditions replicated those of Darwin and Baddeley. Namely, dissimilar vocabularies of both durations displayed significant recency and suffix effects, while vocabularies of $60-\mathrm{msec}$ similar syllables exhibited neither of the effects. However, contrary to predicted results based on the tape-recorder model, significant recency and suffix effects were found for $190-\mathrm{msec}$ similar syllables. Further studies have also demonstrated that stimulus duration has a significant effect on the subject's performance. Fujisaki and Kawashima (1969) have shown that listeners' reliance on category assignment for discrimination increases as the duration of synthetic vowels is reduced from 6 to 3 to 1 glottal pulses. They proposed that the relatively long-duration-vowel steady-state cues were preserved better than the brief consonant cues. Another study (Crowder, 1973a) suggests that PAS holds steady-state speech sounds for longer time periods than it does transient speech sounds.

Both the present study and that of Darwin and Baddeley have demonstrated that the acoustic distance between the items in the stimulus set has an effect on their preservation in PAS. It is apparent, however, that this stimulus characteristic is not the only determining factor. Stimulus duration is an integral dimension in the determination of an item's preservation in PAS.

In order to account for these results, it is necessary 

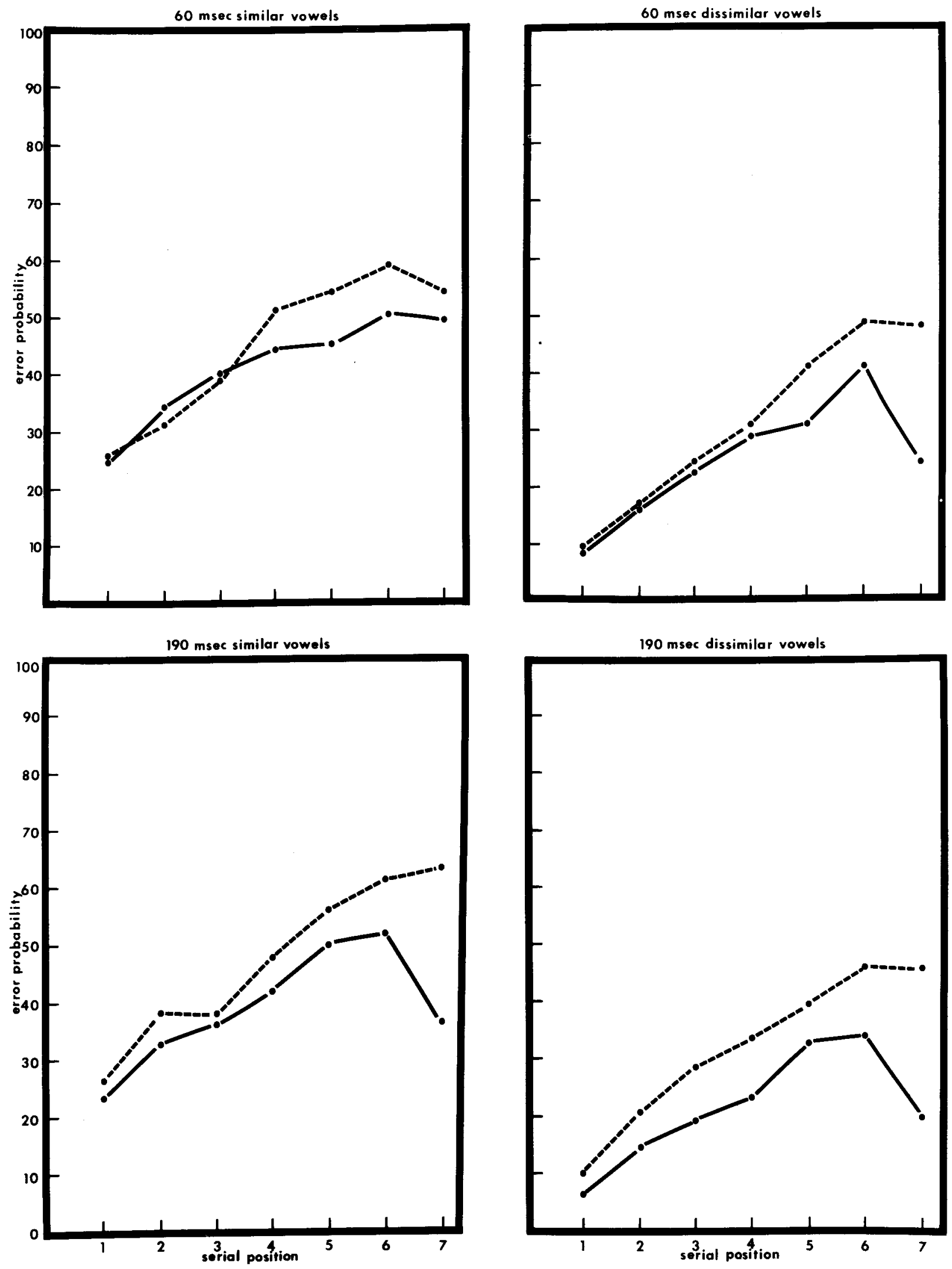

Figure 2. Error probability as a function of serial position for the four vocabularies used. Solid lines represent vocabularies without a suffix and dotted lines represent those with a suffix. 
to postulate a model in which the acoustic properties of the stimulus set work together to produce an integral unit with its own distinguishing characteristics. Some of these acoustic properties include stimulus duration, the acoustic distance between the stimulus items, the onset characteristics of the stimuli (Crowder, 1973b), and other factors which may not as yet have been determined. In particular, with regard to the present study, although many stimulus characteristics may be involved in vocabularies of dissimilar syllables, the acoustic distance between the stimulus items determined the extent to which these vocabularies would be preserved in PAS. For these acoustically distinct stimuli, there is sufficient information remaining after degradation to preserve the items in PAS regardless of stimulus length. In contrast, because similar vocabularies are acoustically less distinct, there is less information available in PAS after degradation to distinguish among the members of the stimulus set. In this case, the absolute duration of the stimuli enhances their perception. When they are relatively short (e.g., $60 \mathrm{msec}$ ), the contribution made by stimulus duration is not sufficient to preserve the sounds in PAS. However, longer durations (e.g., $190 \mathrm{msec}$ ), although not affecting the acoustic distance among the stimuli, provide a greater amount of this information and, as a consequence, facilitate the preservation of these stimuli in PAS.

The fact that the shapes of the memory functions change as a consequence of either the acoustic distance between the stimuli or the duration of the stimulus items when the phonetic characteristics remain identical suggests that PAS is an active perceptual system sensitive to the particular acoustic properties intrinsic to the stimuli. If PAS were basically a passive system, one would expect identical memory curves for all vocabularies regardless of the acoustic characteristics of the stimuli.

It is clear that the ability to distinguish syllables from a given vocabulary is dependent not only on the acoustic distance separating the stimuli, but also on stimulus duration. Any model attempting to explain precategorical acoustic storage must be expanded to account for this phenomenon. Further study is required to determine the variation of this effect as well as any other possible effects caused by altering other, as yet uninvestigated, parameters.

\section{REFERENCES}

CRowder, R. G. Precategorical acoustic storage for vowels of short and long duration. Perception \& Psychophysics, 1973, 3, 502-506. (a)

CROWDER, R. G. Representation of speech sound in precategorical acoustic storage. Journal of Experimental Psychology, 1973, 1. 14-24. (b)

Crowder, R. G. The sound of vowels and consonants in immediate memory. Journal of Verbal Learning and Verbal Behavior. 1971. 10. 587-596.

Crowder, R. G., \& Morton, J. Precategorical acoustic storage (PAS). Perception \& Psychophysics, 1969, 5, 365-373.

Darwin, C. J., \& Baddeley, A. D. Acoustic memory and the perception of speech. Cognitive Psychology, 1974, 6, 41-60.

Fujisaki, H., \& Kawashima, T. On the modes and mechanisms of speech perception. Annual Report of the Engineering Research Institute (University of Tokyo), 1969. 28. 767.773.

\section{NOTE}

1. Previous studies have shown that a tone suffix has no effect on recall of the stimulus list. Therefore, presentation of lists without an eighth syllable suffix ending was considered to be equivalent to the tone control used formerly.

(Received for publication December 20, 1976; accepted for publication April 27, 1977.) 